\title{
函数域中的 $L_{1}$ 范数堆积
}

\author{
李鸿利 ${ }^{*}$ \\ (中国科学技术大学数学系, 合肥 230026)
}

摘要讨论方体中的堆积 (packing), 即如何在方体中放置 $n$ 个点, 使得它们之 间的最短距离最大. 使用的距离是由 $L_{1}$ 范数诱导出来的, 这正类似于纠错码中 常用的 Hamming 距离. 利用函数域中关于除子类群等的研究成果, 给出了两个 具有合理参数的堆积的构造方法, 同时还给出了这两个堆积的一些近似结果.

\section{关键词 $L_{1}$ 范数 堆积 (packing) 亏格 类数 纠错码}

\section{1 引言}

经典的球体堆积 (packing) 问题研究的是: 如何在 $n$ 维 Euclid 空间中放置一 些互相不重叠的小球体, 使得堆积密度达到最大, 这个问题至今仍未能完全解 决. 这里的堆积密度指的是球体在空间中所占的体积的比例. 著名的 Kepler 问题 (Kepler 猜想) 就是寻找 3 维 Euclid 空间中的最大密度的堆积问题. 另一个令人关 注的问题是吻数 (kissing number) 问题, $n$ 维吻数指的是, 在 $n$ 维 Euclid 空间 $\mathbb{R}^{n}$ 中的一个球体外放置互不重叠的与此球相切的相同球体的最大个数. Newton十 三球猜想说的是 3 维吻数为 13 , 而现已证明 3 维吻数为 12 .

吻数问题又可以描述为: $n$ 维空间 $\mathbb{R}^{n}$ 中的一个球面上最多可以放置多少个 点, 使得任何两点与球心之间的夹角至少为 $\frac{\pi}{3}$, 因此吻数问题也可以认为是一个 堆积问题, 即 $\mathbb{R}^{n}$ 中的球面 (而不是 $\mathbb{R}^{n}$ ) 上的堆积问题. 我们还可以作如下更为 重要的推广: 如何在球体中、方体中或者 $\mathbb{R}^{n}$ 的任何子集中放置点, 使得最短距 离达到最大. 毫无疑问纠错码和堆积之间有着紧密的联系, 因为纠错码也就是 $\mathbb{F}_{q}^{n}$ (而不是 $\mathbb{R}^{n}$ ) 中的堆积. 如果想进一步地了解球体堆积, 我们推荐 Conway 和 Sloane 的著作 ${ }^{[1]}$. 本文讨论方体中的堆积, 我们称之为方体堆积.

人们一般是在 Euclid 空间中研究堆积问题, 而且采取的方法大多是组合数

2004-11-30 收稿

*E-mail: hlli@ustc.edu 
学、线性代数等等. 本文中, 我们利用函数域的结果给出两个堆积的构造方法, 而且我们做的是 $L_{1}$ 范数空间中的堆积, 这和编码理论有着更为紧密的联系. 两 种构造方法稍微有点不同: 第 1 种可以认为是 Goppa 几何码的一个推广, 而第 2 种和函数域的除子类群相关联. 但是, 两种构造方法都将给出参数合理的堆积.

以下给出一些必要的定义:

定义 1.1 若 $x=\left(x_{1}, \cdots, x_{n}\right) \in \mathbb{R}^{n}$, 则 $x$ 的 $L_{1}$ 范数定义为 $|x|_{1}:=\sum_{i=1}^{n}\left|x_{i}\right|$, 记作 $|x|_{1}$. 对另一个 $y=\left(y_{1}, \cdots, y_{n}\right) \in \mathbb{R}^{n}, x$ 和 $y$ 之间的距离定义为 $d(x, y):=$ $|x-y|_{1}=\sum_{i=1}^{n}\left|x_{i}-y_{i}\right|$, 记为 $d(x, y)$.

若 $S$ 是 $\mathbb{R}^{n}$ 的一个子集, $S$ 的最小距离定义为 $d(S):=\min \{d(x, y) \mid x, y \in$ $S, x \neq y\}$.

定义 1.2 一个 $(n, M, d)$ 方体堆积指的是 $I^{n}:=[0,1]^{n} \subseteq \mathbb{R}^{n}$ 中的一个包含 $M$ 个元素, 且最小距离不小于 $d$ 的子集合.

\section{2 函数域背景}

在这一节中, 我们假设 $k$ 是任意的一个域. 我们将不加证明地给出代数函 数域中的一些基本定义和结果, 具体细节可参见文献 [2].

定义 2.1 $F$ 是 $k$ 的一个扩域, 且对于 $k$ 上的一个超越元 $x \in F, F$ 是 $k(x)$ 的有限代数扩张, 则 $F$ 是 $k$ 上的一个代数函数域, 记作 $F / k$.

$k$ 上的代数函数域 $F$ 的一个正规化离散赋值是 $F \rightarrow \mathbb{Z} \cup\{\infty\}$ 的一个满射 $v$, 满足:

(1) $v(x)=0$ 当且仅当 $x=\infty$;

(2) $v(x y)=v(x)+v(y)$, 对任意的 $x, y \in F$;

(3) $v(x+y) \leqslant \min (v(x), v(y))$, 对任意的 $x, y \in F$;

(4) $v(a)=0$, 对所有 $a \in k^{*}$.

$F$ 的一个位 $P$ 是 $F$ 的某个赋值环的极大理想. 我们把位 $P$ 对应的赋值环记 为 $O_{P} . F$ 的位和 $F$ 的正规化离散赋值之间有个一一对应, 把 $F$ 的位 $P$ 对应的正 规化离散赋值记作 $v_{P}$, 则

$$
O_{P}=\left\{x \in F: v_{P}(x) \geqslant 0\right\}, \quad P=\left\{x \in O_{P}: v_{P}(x)>0\right\},
$$

$P$ 对应的单位群 $U_{P}$ 定义为

$$
U_{P}=\left\{x \in O_{P}: v_{P}(x)=0\right\} .
$$

商域 $O_{P} / P$ 记作 $F_{P}$, 称为 $P$ 的剩余类域, 它可以自然地看作 $k$ 的一个有限 扩张. 我们称 $F_{P}$ 的扩张次数 $\left[F_{P}: k\right]$ 为 $P$ 的度, 记为 $\operatorname{deg}(P)$. 度为 1 的位称为 有理位. 记 $\mathbf{P}_{F}$ 为 $F$ 的所有位组成的集合, 我们有

定理 2.2 (逼近定理) 设 $S$ 是 $\mathbf{P}_{F}$ 的一个非空真子集, $P_{1}, \cdots, P_{r} \in S$, 则对 任意给定的 $F$ 中的元素 $x_{1}, \cdots, x_{r} \in F$ 和给定的整数 $n_{1}, \cdots, n_{r} \in \mathbb{Z}$, 存在 $x \in F$, 使得 $v_{P_{i}}\left(x-x_{i}\right)=n_{i}(i=1, \cdots, r)$, 且对任意 $P \in S \backslash\left\{P_{1}, \cdots, P_{r}\right\}$, 有 $v_{P}(x) \geqslant 0$.

$P$ 为 $F$ 的位, 如果 $t \in F$ 满足 $v_{P}(t)=1$, 则称 $t$ 为 $P$ 的一个局部化参数. 我 
们有在 $P$ 位的局部展开:

定理 2.3 设 $t$ 为 $P$ 的一个局部化参数, 则任意的 $f \in F$ 可以惟一地展开成

$$
f=\sum_{i=r}^{\infty} a_{i} t^{i}
$$

其中 $a_{i} \in F_{P}, a_{r} \neq 0$, 且 $v_{P}(f)=r$.

$F$ 的除子群 $\operatorname{Div}(F)$ 是由 $F$ 的所有位生成的自由 Abel 群:

$$
\operatorname{Div}(F)=\left\{\sum_{P \in S} m_{P} P: S \text { 是 } \mathbf{P}_{F} \text { 的有限子集，且对任意的 } P \in S, \text { 有 } m_{P} \in \mathbb{Z}\right\} \text {. }
$$

$F$ 的一个除子 $D$ 是如下的一个形式和:

$$
D=\sum_{P \in \mathbf{P}_{F}} m_{P} P
$$

其中 $m_{P}$ 为整系数, 且对至多有限个 $P \in \mathbf{P}_{F}$ 有 $m_{P} \neq 0$. 我们记 $v_{P}(D)$ 为 $D$ 在 $P$ 位的系数 $m_{P}$, 则 $D$ 的支撑集 $\operatorname{supp}(D)$ 是 $\mathbf{P}_{F}$ 的一个有限子集:

$$
\operatorname{supp}(D)=\left\{P \in \mathbf{P}_{F}: v_{P}(D) \neq 0\right\} .
$$

$D$ 的度 $\operatorname{deg}(D)$ 定义为

$$
\operatorname{deg}(D)=\sum_{P \in \operatorname{supp}(D)} v_{P}(D) \operatorname{deg}(P) .
$$

对于 $F$ 的两个除子 $D_{1}$ 和 $D_{2}$, 如果对任意 $P \in \mathbf{P}_{F}$ 有 $v_{P}\left(D_{1}\right) \leqslant v_{P}\left(D_{2}\right)$, 则称 $D_{1} \leqslant D_{2}$. 特别地, 如果 $D \geqslant 0$, 则称 $D$ 为正除子.

任给 $x \in F^{*}$, 定义 $x$ 的零除子

$$
(x)_{0}=\sum_{v_{P}(x)>0} v_{P}(x) P
$$

和 $x$ 的极除子

$$
(x)_{\infty}=\sum_{v_{P}(x)<0}\left(-v_{P}(x)\right) P
$$

$x$ 的主除子为

$$
\operatorname{div}(x)=(x)_{0}-(x)_{\infty} .
$$

任意的主除子的度均为 0 , 即

$$
\operatorname{deg}\left((x)_{0}\right)=\operatorname{deg}\left((x)_{\infty}\right) .
$$

$F$ 的一个除子 $D$ 的 Riemann-Roch 空间定义为

$$
\mathcal{L}(D)=\left\{x \in F^{*}: \operatorname{div}(x)+D \geqslant 0\right\} \cup\{0\},
$$

$\mathcal{L}(D)$ 是 $k$ 上的一个有限维线性空间，记这个空间的维数为 $\operatorname{dim}(D)$, 称之为 $D$ 的 维数. 
定理 2.4 (Riemann-Roch 定理) 若 $F$ 是一个亏格为 $g$ 的代数函数域, 则对任 意的除子 $D$ 有

$$
\operatorname{dim}(D) \geqslant \operatorname{deg}(D)-g+1,
$$

当 $\operatorname{deg}(D) \geqslant 2 g-1$ 时等式成立.

再看除子群的定义, 记 $\operatorname{Div}^{0}(F)$ 是 $F$ 的所有度为 0 的除子组成的集合, 容易 验证它构成一个群, 而且是 $\operatorname{Div}(F)$ 的子群. 称 $\operatorname{Div}^{0}(F)$ 是 $F$ 的零除子群.

因为对任意的 $x \in F^{*}$ 都有 $\operatorname{deg}(\operatorname{div}(x))=0$, 因此有 $\operatorname{Div}^{0}(F)$ 的一个子群

$$
\operatorname{Princ}(F)=\left\{\operatorname{div}(x): x \in F^{*}\right\},
$$

记商群 $\operatorname{Div}^{0}(F) / \operatorname{Princ}(F)$ 为 $\mathrm{Cl}(F)$, 称之为 $F$ 的零除子类群, 这是一个有限群, 称它的阶数为 $F$ 的类数, 记作 $h(F)$.

\section{3 堆积的构造}

\section{1 第 1 种构造}

设 $q$ 是一个素数幂, $\mathbb{F}_{q}$ 是 $q$ 元有限域. 我们先给定一些假设:

$F$ 是 $\mathbb{F}_{q}$ 上的一个亏格为 $g$ 的代数函数域, 记为 $F / \mathbb{F}_{q}$.

$P_{1}, \cdots, P_{n}$ 是 $F$ 的 $n$ 个互不相同的有理位.

$G=P_{1}+\cdots+P_{n}$.

$D$ 是 $F$ 的一个除子, 且 $\operatorname{supp}(D) \bigcap \operatorname{supp}(G)=\emptyset$.

$\theta$ 是 $\mathbb{F}_{q}$ 到 $\{0,1, \cdots, q-1\}$ 的一个一一映射, 且 $\theta(0)=0$.

定理 3.1 设 $q$ 是一个素数幂, $k, m$ 是满足 $m \geqslant 1, m n-k>0$ 的整数, 则 存在一个 $(n, M, d)$ 方体堆积, 使得

$$
M \geqslant q^{m n-k-g+1}, \quad d \geqslant \frac{k}{m q^{m}} .
$$

证 设 $D$ 是一个除子且 $\operatorname{deg}(D)=m n-k(m \geqslant 1) . t_{i}$ 是 $P_{i}$ 的局部化参数, 则任意的 $f \in \mathcal{L}(D)$ 可以展开成

$$
f=\sum_{j=0}^{\infty} a_{i j} t_{i}^{j}
$$

考虑映射

$$
\begin{gathered}
\pi: \mathcal{L}(D) \rightarrow \mathbb{R}^{n}, \\
f \mapsto \pi(f):=\left(\sum_{j=0}^{m-1} \frac{\theta\left(a_{1 j}\right)}{q^{j+1}}, \cdots, \sum_{j=0}^{m-1} \frac{\theta\left(a_{n j}\right)}{q^{j+1}}\right),
\end{gathered}
$$

因为

$$
\left|\sum_{j=0}^{m-1} \frac{\theta\left(a_{i j}\right)}{q^{j+1}}\right| \leqslant(q-1) \sum_{j=0}^{m-1} \frac{1}{q^{j+1}}=1-\frac{1}{q^{m}}<1 \quad(i=1, \cdots, n),
$$

所以 $\pi(f) \in I^{n}$. 
任意 $f, g \in \mathcal{L}(\mathcal{D}), f=\sum_{j=0}^{\infty} a_{i j} t_{i}^{j}, g=\sum_{j=0}^{\infty} b_{i j} t_{i}^{j}, f \neq g, \pi(f)-\pi(g)=$ $\left(c_{1}, \cdots, c_{n}\right)$, 其中 $c_{i}=\sum_{j=0}^{m-1} \frac{\theta\left(a_{i j}\right)-\theta\left(b_{i j}\right)}{q^{j+1}}$, 假设刚好有 $t$ 个坐标 $c_{i_{1}}, c_{i_{2}}, \cdots, c_{i_{t}}$ 等 于 0 (这就意味着 $v_{P_{i_{s}}}(f-g) \geqslant m(s=1, \cdots, t)$ ), 则

$$
0 \neq f-g \in \mathcal{L}\left(D-m\left(P_{i_{1}}+P_{i_{2}}+\cdots+p_{i_{t}}\right)\right)
$$

从而有

$$
0 \leqslant \operatorname{deg}\left(D-m\left(P_{i_{1}}+P_{i_{2}}+\cdots+p_{i_{t}}\right)\right)=\operatorname{deg}(D)-m t
$$

所以 $t \leqslant n-\frac{k}{m}$.

如果 $c_{i} \neq 0$, 则一定存在某个整数 $r$, 使得

$$
\left|c_{i}\right|=\left|\sum_{j=0}^{m-1} \frac{\theta\left(a_{i j}\right)-\theta\left(b_{i j}\right)}{q^{j+1}}\right| \geqslant \frac{1}{q^{r}}-\left(\frac{q-1}{q^{r+1}}+\cdots+\frac{q-1}{q^{m}}\right)=\frac{1}{q^{m}},
$$

从而 $|\pi(f)-\pi(g)|_{1} \geqslant(n-t) \frac{1}{q^{m}} \geqslant \frac{k}{m} \cdot \frac{1}{q^{m}}$, 即 $d \geqslant \frac{k}{m} \cdot \frac{1}{q^{m}}$.

另一方面, 由 Riemann-Roch 定理,

$$
\operatorname{dim}(D) \geqslant \operatorname{deg}(D)-g+1=m n-k-g+1,
$$

所以有 $M \geqslant q^{m n-k-g+1}$. 定理得证.

注 当 $m=1$ 时, 这个堆积的构造完全类似 Goppa 几何码, 而且最小距离 可以改进为 $k /(q-1)$, 只需要在前面的证明中考虑映射

$$
\begin{gathered}
\pi: \mathcal{L}(D) \rightarrow \mathbb{R}^{n}, \\
f \mapsto \pi(f):=\left(\frac{\theta\left(a_{10}\right)}{q-1}, \cdots, \frac{\theta\left(a_{n 0}\right)}{q-1}\right)
\end{gathered}
$$

即可.

\section{2 第 2 种构造}

在介绍这种构造前, 还需要介绍一些关于函数域的定义和结果. 设 $q$ 是一 个素数幂, $\mathbb{F}_{q}$ 是 $q$ 元有限域, $F / \mathbb{F}_{q}$ 是一个方格为 $g$, 类数是 $h$ 的代数函数域. 接 下来我们将取定一个正除子 $D=\sum_{P} m_{P} P$. 对任意 $f \in F^{*}$, 称

$$
f \equiv 1 \quad(\bmod D)
$$

如果 $f$ 满足以下条件: 对任意 $P \in \operatorname{supp}(D), f \in O_{P}$ 且 $v_{P}(f-1) \geqslant v_{p}(D)$. 显 然, 如果 $f \equiv 1(\bmod D)$, 则对任意 $P \in \operatorname{supp}(D)$, 一定有 $v_{P}(f)=0$.

考虑 $F$ 的零除子群 $\operatorname{Div}^{0}(F)$ 的子群

$$
\operatorname{Div}_{D}^{0}(F):=\left\{G \in \operatorname{Div}^{0}(F): \operatorname{supp}(G) \cap \operatorname{supp}(D)=\emptyset\right\},
$$

再定义 $\operatorname{Div}_{D}^{0}(F)$ 的子群

$$
U_{D}(F):=\left\{\operatorname{div}(f): f \in F^{*}, \operatorname{supp}(\operatorname{div}(f)) \cap \operatorname{supp}(D)=\emptyset\right\},
$$

可以验证 $U_{D}(F)$ 有如下子群:

$$
\operatorname{Princ}_{D}(F):=\left\{\operatorname{div}(f): f \in F^{*}, f \equiv 1(\bmod D)\right\} .
$$


把商群 $\operatorname{Div}_{D}^{0}(F) / \operatorname{Princ}_{D}(F)$ 记作 $\mathrm{Cl}_{D}(F)$, 并且称之为模 $D$ 的狭窄射线类群 (narrow ray class group).

引理 3.2 $\operatorname{Div}_{D}^{0}(F) / U_{D}(F)$ 同构于 $\mathrm{Cl}(F)$, 其中 $\mathrm{Cl}(F)$ 是 $F$ 的零除子类群.

证 设 $G$ 为 $\mathrm{Cl}(F)$ 中的任意一个元素, 由逼近定理知, 存在 $f \in F^{*}$, 使得 对任意的 $P \in \operatorname{supp}(D)$,

$$
v_{P}(f)=v_{P}(G)
$$

所以 $G=G-\operatorname{div}(f) \in \operatorname{Cl}(F)$, 而且有 $G-\operatorname{div}(f) \in \operatorname{Div}_{D}^{0}(F)$.

考虑映射

$$
\begin{aligned}
\Phi: \mathrm{Cl}(F) & \rightarrow \operatorname{Div}_{D}^{0}(F) / U_{D}(F), \\
G & \mapsto G-\operatorname{div}(f),
\end{aligned}
$$

容易验证 $\Phi$ 是一个合理定义的满同态. 如果 $\Phi\left(G_{1}\right)=\Phi\left(G_{2}\right)$, 则存在 $g \equiv 1$ $(\bmod D)$ 和 $f_{1}, f_{2} \in F^{*}$, 使得 $G_{1}-G_{2}=\operatorname{div}\left(\left(g f_{1}\right) / f_{2}\right)$, 从而在 $\operatorname{Cl}(F)$ 中有 $G_{1}=G_{2}$, 即 $\Phi$ 是单射. 所以 $\Phi$ 是一个同构. 证毕.

对 $F$ 的一个位 $P$ 和正整数 $n \geqslant 1$, 定义 $P$ 位的 $n$ 次单位群如下:

$$
U_{P}^{(n)}:=\left\{f \in U_{P}: v_{P}(f-1) \geqslant n\right\},
$$

其中 $U_{P}$ 是 $P$ 的单位群.

引理 $3.3 F_{P}$ 为 $P$ 的类域, 则

(1) $U_{P} / U_{P}^{(1)} \cong F_{P}^{*}$;

(2) $U_{P}^{(n)} / U_{P}^{(n+1)} \cong F_{P}^{+}$.

引理的证明可参见文献 [3].

\section{引理 3.4}

$$
U_{D}(F) / \operatorname{Princ}_{D}(F) \cong\left(\prod_{P \in \operatorname{supp}(D)} U_{P} / U_{P}^{\left(m_{P}\right)}\right) / \mathbb{F}_{q}^{*},
$$

其中 $D=\sum_{P} m_{P} P$.

证 定义

$$
\begin{aligned}
\Phi: U_{D}(F) & \rightarrow\left(\prod_{P \in \operatorname{supp}(D)} U_{P} / U_{P}^{\left(m_{P}\right)}\right) / \mathbb{F}_{q}^{*}, \\
\operatorname{div}(x) & \mapsto(x, \cdots, x) .
\end{aligned}
$$

对任意的 $\operatorname{div}(x), \operatorname{div}(y) \in U_{D}(F), \operatorname{div}(x)=\operatorname{div}(y)$ 当且仅当存在 $\lambda \in \mathbb{F}_{q}^{*}$, 使得 $x=\lambda y$, 从而 $\Phi(\operatorname{div}(x))=\Phi(\operatorname{div}(y))$. 所以 $\Phi$ 的定义是合理的.

$\operatorname{div}(x) \in \operatorname{Ker} \Phi$ 当且仅当存在 $\lambda \in \mathbb{F}_{q}^{*}$, 使得对任意的 $P \in \operatorname{supp}(D)$, 有 $\lambda x \in$ $U_{P}^{\left(m_{P}\right)}$, 即对任意 $P \in \operatorname{supp}(D), v_{P}(\lambda x-1) \geqslant m_{P}$. 由 $\operatorname{Princ}_{D}(F)$ 的定义可知 $\operatorname{div}(x)=\operatorname{div}(\lambda x) \in \operatorname{Princ}_{D}(F)$, 从而有

$$
\operatorname{Ker} \Phi=\operatorname{Princ}_{D}(F) .
$$


任取 $\left(x_{P}\right)_{P} \in\left(\prod_{P \in \operatorname{supp}(D)} U_{P} / U_{P}^{\left(m_{P}\right)}\right) / \mathbb{F}_{q}^{*}$, 由逼近定理, 存在 $x \in F^{*}$, 使得对任 意 $P \in \operatorname{supp}(D)$,

$$
v_{P}\left(x-x_{P}\right) \geqslant m_{P},
$$

这意味着 $x \in U_{P}$ 且对任意 $P \in \operatorname{supp}(D)$, 在 $U_{P} / U_{P}^{\left(m_{P}\right)}$ 中有 $x=x_{P}$, 由此即得 $\operatorname{div}(x) \in U_{D}(F)$ 且 $\Phi(\operatorname{div}(x))=\left(x_{P}\right)_{P}$, 从而 $\Phi$ 是满射.

由同态基本定理有 $U_{D}(F) / \operatorname{Ker} \Phi \cong \operatorname{Im} \Phi$, 再由 $\operatorname{Ker} \Phi=\operatorname{Princ}_{D}(F)$ 和 $\Phi$ 是满 射即得引理. 证毕.

由引理 3.3 得到

$$
\begin{aligned}
\left|U_{P} / U_{P}^{\left(m_{P}\right)}\right| & =\left|U_{P} / U_{P}^{(1)}\right| \cdot\left|\prod_{i=2}^{m_{P}} U_{P}^{(i-1)} / U_{P}^{(i)}\right|=\left|F_{P}^{*}\right| \cdot\left|\prod_{i=2}^{m_{P}} F_{P}^{+}\right| \\
& =\left(q^{\operatorname{deg}(P)}-1\right) q^{\left(m_{P}-1\right) \operatorname{deg}(P)},
\end{aligned}
$$

再由引理 3.2 和 3.4 , 有

$$
\begin{aligned}
\left|\mathrm{Cl}_{D}(F)\right| & =\left|\operatorname{Div}_{D}^{0}(F) / \operatorname{Princ}_{D}(F)\right|=\left|\operatorname{Div}_{D}^{0}(F) / U_{D}(F)\right| \cdot\left|U_{D}(F) / \operatorname{Princ}_{D}(F)\right| \\
& =|\operatorname{Cl}(F)| \cdot\left|\left(\prod_{P \in \operatorname{supp}(D)} U_{P} / U_{P}^{\left(m_{P}\right)}\right) / \mathbb{F}_{q}^{*}\right| \\
& =\frac{h}{q-1} \cdot \prod_{m_{P} \geqslant 1}\left(q^{\operatorname{deg}(P)}-1\right) q^{\left(m_{P}-1\right) \operatorname{deg}(P)} .
\end{aligned}
$$

定理 3.5 设 $P_{1}, \cdots, P_{n}$ 是 $F / \mathbb{F}_{q}$ 的 $n$ 个不同有理位, $D=\sum_{P} m_{P} P$ 是满足 $\left\{P_{1}, \cdots, P_{n}\right\} \cap \operatorname{supp}(D)=\emptyset$ 的正除子, 则对任意正整数 $r$, 存在一个 $(n, M, d)$ 方 体堆积, 满足

$$
M \geqslant \frac{(q-1)(r+1)^{n}}{h \cdot \prod_{m_{P} \geqslant 1}\left(q^{\operatorname{deg} P}-1\right) q^{\left(m_{P}-1\right) \operatorname{deg} P}}, \quad d \geqslant \frac{\operatorname{deg} D}{r} .
$$

证 考虑映射

$$
\begin{aligned}
\pi:\left\{\frac{0}{r}, \frac{1}{r}, \cdots, \frac{r-1}{r}, \frac{r}{r}\right\}^{n} & \rightarrow \mathrm{Cl}_{D}(F), \\
\left(c_{1}, \cdots, c_{n}\right) & \mapsto \sum_{i=1}^{n}\left(r c_{i}\right) P_{i},
\end{aligned}
$$

因为 $\mathrm{Cl}_{D}(F)$ 中只有 $H:=\frac{h}{q-1} \cdot \prod_{m_{P} \geqslant 1}\left(q^{\operatorname{deg}(P)}-1\right) q^{\left(m_{P}-1\right) \operatorname{deg}(P)}$ 个元素, 所以 可在 $\mathrm{Cl}_{D}(F)$ 中找到一个元素 $G$, 使得它至少有 $(r+1)^{n} / H$ 个原像. 把 $G$ 的原像 集记作

$$
C=\pi^{-1}(G)
$$

则 $|C| \geqslant(r+1)^{n} / H$. 要证 $C$ 是一个满足所给参数的 $(n, M, d)$ 方体堆积, 只需要 证明 $C$ 的最小距离至少是 $\operatorname{deg} D / r$.

设 $u=\left(u_{1}, \cdots, u_{n}\right), v=\left(v_{1}, \cdots, v_{n}\right)$ 是 $C$ 中两个不同的元素，则 $\pi(u)=$ $\pi(v)=G$, 从而 $\pi(u)-\pi(v)$ 是 $\mathrm{Cl}_{D}(F)$ 中的零元素, 即 $\pi(u)-\pi(v) \in \operatorname{Princ}_{D}(F)$. 
存在 $f \in F^{*}$, 使得

$$
\operatorname{div}(f)=\pi(u)-\pi(v)=\sum_{u_{i}>v_{i}} r\left(u_{i}-v_{i}\right) P_{i}-\sum_{u_{i}<v_{i}} r\left(v_{i}-u_{i}\right) P_{i}
$$

且

$$
f \equiv 1 \quad(\bmod D)
$$

故有

$$
(f)_{0}=\sum_{u_{i}>v_{i}} r\left(u_{i}-v_{i}\right) P_{i}, \quad(f)_{\infty}=\sum_{u_{i}<v_{i}} r\left(v_{i}-u_{i}\right) P_{i},
$$

其中 $(f)_{0}$ 和 $(f)_{\infty}$ 分别是 $f$ 的零除子和极除子.

$f \equiv 1(\bmod D)$ 意味着 $D \leqslant(f-1)_{0}$, 从而

$$
\begin{aligned}
\operatorname{deg}(D) & \leqslant \operatorname{deg}(f-1)_{0}=\operatorname{deg}(f-1)_{\infty}=\operatorname{deg}(f)_{\infty} \\
& =\operatorname{deg}\left(\sum_{u_{i}<v_{i}} r\left(v_{i}-u_{i}\right) P_{i}\right)=\sum_{u_{i}<v_{i}} r\left(v_{i}-u_{i}\right),
\end{aligned}
$$

即得

$$
d(u, v)=\sum_{i=1}^{n}\left|u_{i}-v_{i}\right| \geqslant \frac{1}{r} \sum_{u_{i}<v_{i}} r\left(v_{i}-u_{i}\right) \geqslant \frac{1}{r} \cdot \operatorname{deg} D .
$$

定理证毕.

\section{3 近似结果}

在编码理论中，构造好的码字面临的一个问题是: 对给定的 $q$ 和相对距离 $d / n$, 如何寻找 $\mathbb{F}_{q}$ 上的 $(n, M, d)$ 码, 使得它的信息率 $\log M / n$ 尽可能达到最大. 类似地, 在方体堆积中也有这样的问题: 对给定的 $d / n$, 寻找 $(n, M, d)$ 方体堆积, 使得 $\log M / n$ 尽可能达到最大. 近似问题就是在 $n$ 非常大的情形下来处理这个 问题的.

$U$ 是 $(0,1] \times[0,+\infty)$ 的一个子集合, 其中的元素 $(\delta, R)$ 满足: 存在一个 $\left(n_{i}, M_{i}, d_{i}\right)$ 方体堆积序列 $(i=1,2, \cdots)$, 当 $i \rightarrow \infty$ 时, $n_{i} \rightarrow \infty$, 而且

$$
\delta=\lim _{i \rightarrow \infty} \frac{d_{i}}{n_{i}}, \quad R=\lim _{i \rightarrow \infty} \frac{\log M_{i}}{n_{i}} .
$$

定义 4.1 对任意 $0<\delta \leqslant 1$, 定义

$$
R(\delta)=\sup \{R \in[0,+\infty):(\delta, R) \in U\} .
$$

下面给出从我们的构造中得到的 $R(\delta)$ 的下界:

定义 4.2 对任意 $g \geqslant 0$, 定义

$N_{q}(g)=\sup \left\{N(F): F\right.$ 是 $\mathbb{F}_{q}$ 上的亏格为 $g$ 的代数函数域 $\}$,

其中 $N(F)$ 为 $F / \mathbb{F}_{q}$ 的有理位个数. 再定义 $A(q)$ 为

$$
A(q)=\limsup _{g \rightarrow \infty} \frac{N_{q}(g)}{g} .
$$


关于 $A(q)$ 的界, 读者可以参考文献 [2].

现在我们可以给出从第 1 种构造中得到的 $R(\delta)$ 的下界.

命题 4.3 对任意给定的整数 $m$ 和素数幂 $q$, 有

$$
R(\delta) \geqslant\left(m-m q^{m} \delta-\frac{1}{A(q)}\right) \log q,
$$

其中 $0<\delta \leqslant \frac{1}{m q^{m}}$.

证 设 $F_{i}$ 是 $\mathbb{F}_{q}$ 上的一个函数域序列, 其亏格 $g_{i}$ 和有理位个数 $n_{i}$ 满足

$$
\lim _{i \rightarrow \infty} n_{i}=\infty, \quad \lim _{i \rightarrow \infty} \frac{n_{i}}{g_{i}}=A(q) .
$$

对给定的 $m, q, \delta$, 可以选取 $0 \leqslant k_{i}<n_{i}$, 使得

$$
\lim _{i \rightarrow \infty} \frac{k_{i}}{m q^{m} n_{i}}=\delta .
$$

由定理 3.1, 我们可以得到一个 $\left(n_{i}, q^{m n_{i}-k_{i}-g_{i}+1}, k_{i} /\left(m q^{m}\right)\right)$ 方体堆积序列, 且

$$
\lim _{i \rightarrow \infty} \frac{k_{i}}{m q^{m} n_{i}}=\delta, \quad \lim _{i \rightarrow \infty} \frac{\log q^{m n_{i}-k_{i}-g_{i}+1}}{n_{i}}=\left(m-m q^{m} \delta-\frac{1}{A(q)}\right) \log q .
$$

从 $R(\delta)$ 的定义即可知, 对任意 $0<\delta \leqslant \frac{1}{m q^{m}}$,

$$
R(\delta) \geqslant\left(m-m q^{m} \delta-\frac{1}{A(q)}\right) \log q .
$$

证毕.

设 $F$ 是 $\mathbb{F}_{q}$ 上任意一个亏格为 $g$ 的函数域, 由文献 [4] 中的定理 V.1.15 和 V.2.1, 我们容易得到 $F$ 的类数 $h$ 满足

$$
h \leqslant\left(1+q^{1 / 2}\right)^{2 g} .
$$

再由定理 3.5, 对任意的正除子 $D=\sum_{P} m_{P} P$, 存在一个 $(n, M, d)$ 方体堆积, 满足

$$
\begin{aligned}
M & \geqslant \frac{(q-1)(r+1)^{n}}{h \cdot \prod_{m_{P}} \geqslant 1}\left(q^{\operatorname{deg} P}-1\right) q^{\left(m_{P}-1\right) \operatorname{deg} P} \\
& \geqslant \frac{(q-1)(r+1)^{n}}{h \cdot q^{\operatorname{deg} D}} \geqslant \frac{(q-1)(r+1)^{n}}{\left(1+q^{1 / 2}\right)^{2 g} \cdot q^{\operatorname{deg} D}},
\end{aligned}
$$

且 $d \geqslant \operatorname{deg} D / r$.

下面给出由第 2 种构造得到的界.

命题 4.4 设任意给定的正整数 $r$, 素数幕 $q$ 和正实数 $\delta$, 满足

$$
\delta<\frac{\log (r+1)-(2 / A(q)) \log \left(1+q^{1 / 2}\right)}{r \log q},
$$

则有

$$
R(\delta) \geqslant \log (r+1)-\frac{2}{A(q)} \log \left(1+q^{1 / 2}\right)-r \delta \log q .
$$

证 设 $F_{i}$ 是 $\mathbb{F}_{q}$ 上的一个函数域序列, 其亏格 $g_{i}$ 和有理位个数 $n_{i}$ 满足

$$
\lim _{i \rightarrow \infty} n_{i}=\infty, \quad \lim _{i \rightarrow \infty} \frac{n_{i}}{g_{i}}=A(q) .
$$


对给定的 $r$ 和 $\delta$, 可以选取 $F_{i}$ 的正除子 $D_{i}$, 使得

$$
\lim _{i \rightarrow \infty} \frac{\operatorname{deg} D_{i}}{r n_{i}}=\delta .
$$

由以上的结论和定理 3.5, 可以得到一个 $\left(n_{i}, \frac{(q-1)(r+1)^{n_{i}}}{\left(1+q^{1 / 2}\right)^{2 g_{i}} q^{\mathrm{deg} D_{i}}}, \frac{\operatorname{deg} D_{i}}{r}\right)$ 方体堆积序 列, 且

$$
\begin{gathered}
\lim _{i \rightarrow \infty} \frac{\operatorname{deg} D_{i}}{r n_{i}}=\delta, \\
\lim _{i \rightarrow \infty} \frac{\left.\log (q-1)(r+1)^{n_{i}} /\left(\left(1+q^{1 / 2}\right)^{2 g_{i}} q^{\operatorname{deg} D_{i}}\right)\right)}{n_{i}} \\
=\log (r+1)-\frac{2}{A(q)} \log \left(1+q^{1 / 2}\right)-r \delta \log q .
\end{gathered}
$$

由 $R(\delta)$ 的定义即可得到结果.

致谢 作者衷心感谢邢朝平教授的建议和指导.

\section{参考文献}

1 Conway J H, Sloane N J A. Sphere Packings, Lattices, and Groups. 3rd ed. New York: Springer-Verlag, 1998

2 Niederreter H, Xing C P. Rational Points on Curves over Finite Field: Theory and Applications. London Math Soc Lecture Notes Series, Vol 285. Cambridge: Cambridge University Press, 2001

3 Weiss E. Algebraic Number Theory. New York: McGraw-Hill, 1963

4 Stichtenoth H. Algebraic Function Fields and Codes. Berlin: Springer-Verlag, 1993. 167 169 